\title{
Global screening and extended nomenclature for 230 aphidicolin- inducible fragile sites, including 61 yet unreported ones
}

\author{
KRISTIN MRASEK, CHRISTIANE SCHODER, ANNE-CHRISTIN TEICHMANN, KATJA BEHR, BRITTA FRANZE, \\ KATHLEEN WILHELM, NANCY BLAUROCK, UWE CLAUSSEN, THOMAS LIEHR and ANJA WEISE \\ Institute of Human Genetics and Anthropology, D-07740 Jena, Germany
}

Received November 9, 2009; Accepted January 5, 2010

DOI: 10.3892/ijo_00000572

\begin{abstract}
Since the first description of human fragile sites (FS) more than 40 years ago, a variety of substances were reported to induce chromosomal breaks at non-random, breakage-prone regions. According to information available from human genome browsers aphidicolin, an inhibitor of DNA replication induces 77 of 88 known common FS. However, in the literature additional FS are reported, which are also, at least in part, inducible by aphidicolin. To the best of our knowledge, here we present the first and largest ever done systematic, whole genome-directed and comprehensive screening for aphidicolin-inducible breakage-prone regions. The study was performed on stimulated peripheral blood lymphocytes of 3 unrelated healthy individuals. Twenty-five thousand metaphase spreads were analyzed and overall 22,537 FS located in 230 different loci were recorded. Sixtyone of those FS were never observed before and 52 were already previously reported but not included in genome browsers and yet verified. Interestingly, aphidicolin was able to induce all types of rare and common FS, suggesting that these breakage-prone regions are less dependent on the inducing chemicals than originally supposed. Overall, we provide the first comprehensive genome wide map for FS and studied possible correlations of chromosome length and GTG-banding level with FS-frequency. To handle FS better in future, an extension of the already existing alphabetical nomenclature for FS on single chromosomes is suggested.
\end{abstract}

\section{Introduction}

The first description of human fragile sites (FS) dates back more than 40 years (1), and the term fragile site was introduced by Magenis five years later (2). Since that time fragile sites continued as an active area of research in cytogenetics

Correspondence to: Dr Anja Weise, Institute of Human Genetics and Anthropology, D-07740 Jena, Germany

E-mail: aweise@mti.uni-jena.de

Key words: fragile sites, aphidicolin, nomenclature and their definition and classification was subject for several controversies with the increasing knowledge $(3,4)$. Today FS are understood as specific loci that preferentially exhibit gaps and break on metaphase chromosomes following partial inhibition of DNA synthesis. Rare FS are present in a small proportion of individuals and are inherited in a Mendelian manner. In contrast, common FS are present in all individuals and represent the largest class of FS (5). A number of substances are in use to induce common FS e.g. the bromodeoxyuridine (BrdU) or 5-azacytidine $(5$-azaC) $(5,6)$. Common FS are also induceable by aphidicolin that acts as an inhibitor of DNA polymerase $\alpha$ and $\sigma$ (7). Common FS are part of the normal mammalian chromosome structure. With a frequency of $>5 \%$ in normal population they are usually not associated with disease, except for a possible correlation of some FS with cancer-related breakpoints (e.g. 5,7,8,9) or a molecular cytogenetic co-localization with breakpoints in Fanconi anemia patients (10).

According to the present knowledge, only 77 of 88 common FS listed in human genome browsers like NCBI (http://www.ncbi.nlm.nih.gov/) are induceable by aphidicolin. The three most frequently observed such FS are FRA3B in 3p14, FRA16D in 16q23 and FRAXB in Xp22. Therefore, it is not surprising that they are also part of the up to now 22 molecular characterized common FS: FRA1H, FRA1E, FRA2G, FRA3B, FRA4F, FRA6E, FRA6F, FRA7E, FRA7G, FRA7H, FRA7K, FRA7I, FRA8C, FRA9E, FRA9G, FRA11E, FRA11F, FRA11G, FRA13A, FRA16D, FRA18C and FRAXB. Interestingly, not all common FS are necessarily detectable in only one individual. Additionally, differences in the observable FS-frequency within different individuals are well known $(11,12)$. Besides these 88 official, database-annotated common FS others were observed and reported, including six recently reported new sites, particularly FRA4F (13), FRA7K (14), FRA6H (15), FRA9G (16), FRA13E (15) and FRA18C (17).

Thus, with a systematic genome-wide screening for aphidicolin inducible FS in lymphocytes of 3 unrelated healthy individuals here the question should be solved which regions of the human genome are really breakage prone and to what extent they are affected when applying aphidicolin. Twentyfive thousand metaphase spreads presenting 22,537 break events were analyzed by cytogenetics. As a result, 61 new, by now unreported and unclassified FS were detected and the existence of 52 further FS $(18,19)$ was proven. 


\section{Materials and methods}

Cytogenetics and induction of FS. Peripheral blood lymphocytes from three unrelated, healthy persons with normal female karyotype were studied (proband I, II and III). Proband I and II were Caucasian and proband III Caucasian-Asian in origin. Using cytogenetic standard protocols lymphocyte cultures were incubated for $72 \mathrm{~h}$ at $37^{\circ} \mathrm{C}$ in RPMI medium supplemented with $10 \%$ fetal calf serum and $2 \%$ phytohemoagglutinin. Aphidicolin $(0.2 \mu \mathrm{M})$ dissolved in dimethylsulfoxide was added $24 \mathrm{~h}$ before harvesting (20). Colcemid $(0.04 \mu \mathrm{g} /$ $\mathrm{ml}$ ) was added $2 \mathrm{~h}$ before culture termination. Chromosome preparation was done according to standard protocols (21). Additionally lymphocytes from all three subjects were cultivated without aphidicolin and 100 metaphase spreads were evaluated, each, as negative controls, without finding any spontaneous break events. The chromosomes were banded by diamidinophenylindol (DAPI) and evaluation was done using ISIS software (MetaSystems, Altlussheim, Germany) based inverted DAPI-banding.

The 25,000 analyzed metaphase spreads were categorized according to their band level in 300-350, 400-500, 550-650 and $>650$ bands per haploid karyotype. Fifty-eight percent of the metaphase spreads were arrested at a 400-500 band level, $29 \%$ at $550-650,11 \%$ at $300-350$ and $2 \%$ at a band level of $>650$.

A chromosomal lesion was counted when it appeared as a chromatide or chromosome gap or break according to ISCN 2009 (22). A chromosomal site was determined to be a fragile site when it appeared more than once in this study or was reported before elsewhere in the literature.

\section{Results}

New aphidicolin-induced FS. A total of 25,000 metaphase spreads from three female subjects were analyzed after aphidicolin-induced lymphocyte culture. Overall, cytogenetic analysis revealed 22,537 break-events, i.e. 0.91 breaks per metaphase spread. Two hundred and thirty different FS including 61 until now not reported break-prone regions could be described (Table I, entries in bold, and Figs. 2 and 3). A FS was included here as new in case it was observed in this study at least twice. If a break-event was observed at least once in this study and also previously reported as FS it was also included into the 230 ones described here.

The mean frequency of all FS detected in this study was $0.279 \%$ and only 21 out of 230 detected FS appeared with a frequency over $1 \%$. In contrast 92 specific FS have a frequency between 0.1 and $1 \%$ and the main part of 109 FS is expressed between 0.009 and $0.099 \%$ (Table I). The most frequently observed FS were FRA3B (14.153\%), FRA16D $(7.576 \%)$, FRAXB (5.494\%), FRA2H (3.905\%), FRA1M $(3.333 \%)$ and FRA1I $(2.299 \%)$ whereas FS that were described before and detected only once in this study had a frequency of $0.004 \%$ like FRA8F and FRA17D.

Additionally, single-break events were observed in the current study and not described in literature before. These breaks were not rated as new FS. In detail these were 1p34 (proband II), 2q14.2-14.3 (proband II), 3q23 (proband III), 4q32 (proband II), 15q24 (proband II), 20q13.3 (proband I) and 21q11.2 (proband I). Aphidicolin was able to induce different types of known rare and common FS that were also classified as 5-azacytidine, BrdU, folate sensitive and distamycin type before (Table I).

Frequency of FS per metaphase spread and per haploid band level. Not surprisingly the highest amount of breaks per metaphase were found in the highest band levels (>650: $3-12$; 550-650: 3-8) compared to 300-350 and 400-500 band level with 1-4 and 2-5 breaks per metaphase, respectively.

Frequency of FS in different individuals. The FS-frequencies found in the three different studied individuals are detailed in Table I. Interindividual frequency differences for the expression of FS were found. As typical example for individualspecific FS inducibility results obtained for chromosome 10 are summarized in Fig. 1. Especially proband III expressed the highest rate of chromosome 10-specific FS while proband II showed here an extremely low rate and e.g. no expression of FRA10K. Overall, $41 \%$ of all here detected FS were found in proband I, $38 \%$ in proband III and only $21 \%$ in proband II. Strong FS-expression variation rates were also found e.g. for FRA1I, FRA2J, FRA4C, FRA4H, FRA6B, FRA10F and FRA16C. However, beside these strong variations, there were also sites with practically identical expression in all three studied subjects, such as FRA1A, FRA3C or FRA11C (Table I).

Distribution of FS. As shown in Figs. 2 and 3 the breakprone areas are almost equally distributed along each chromosome. Table II summarizes the number of FS per chromosome and the average interval size in megabases $(\mathrm{Mb})$ between the individual FS. The latter is on average $13.63 \mathrm{Mb}$. The chromosome with the highest number of FS is human chromosome 2 with twenty-one break-prone regions. The number of FS is not correlated with chromosomal size as can be exemplified on chromosome 16, which is the most unstable chromosome with FS every 9.89 Mb. The smallest human chromosomes 21 and 22 show only two FS each and are the most stable chromosomes with an average breaking distance of 23.50 and $25.00 \mathrm{Mb}$ besides the Y chromosome, which was not studied here and has only one FS reported in the literature. Moreover, half of human chromosomes contain a FS at the centomere including chromosomes 1, 2, 3, 5, 6, 8, $11,12,16,17,19$ and X. Breakage-prone regions were also detected in heterochromatic blocks of human chromosome 1, 9 and the Y-chromosome.

Of the FS, $24.5 \%$ are located in GTG dark bands, $62 \%$ in light bands, $5 \%$ in centromeres and $8.5 \%$ at the boarder of light and dark bands. The major part of breaks result from chromatide breaks and only a small portion show chromosome breaks or reunions.

Nomenclature of FS. The nomenclature of FS is chromosomespecific and starts with the abbreviation FRA followed by chromosome number and a capital letter according to the appearance of the FS description from pter to qter, starting from $A$ to $Z$ e.g. FRA1A in 1p36. In accordance with these rules, the here reported new FS and also recently reported FS that were not included in human genome browsers before were 


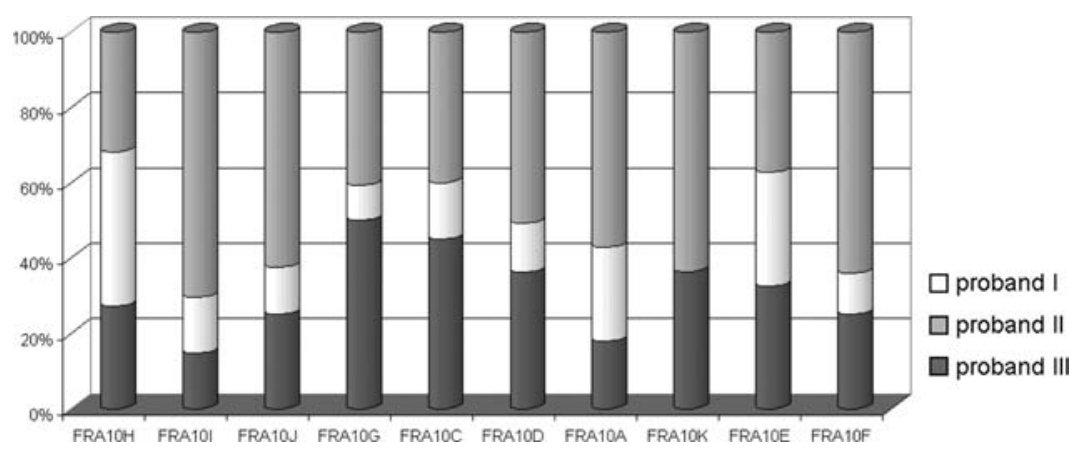

Figure 1. Aphidicolin-induced FS of human chromosome 10 and their frequency in three different individuals (I, II and III). The absolute numbers are given in Table I.

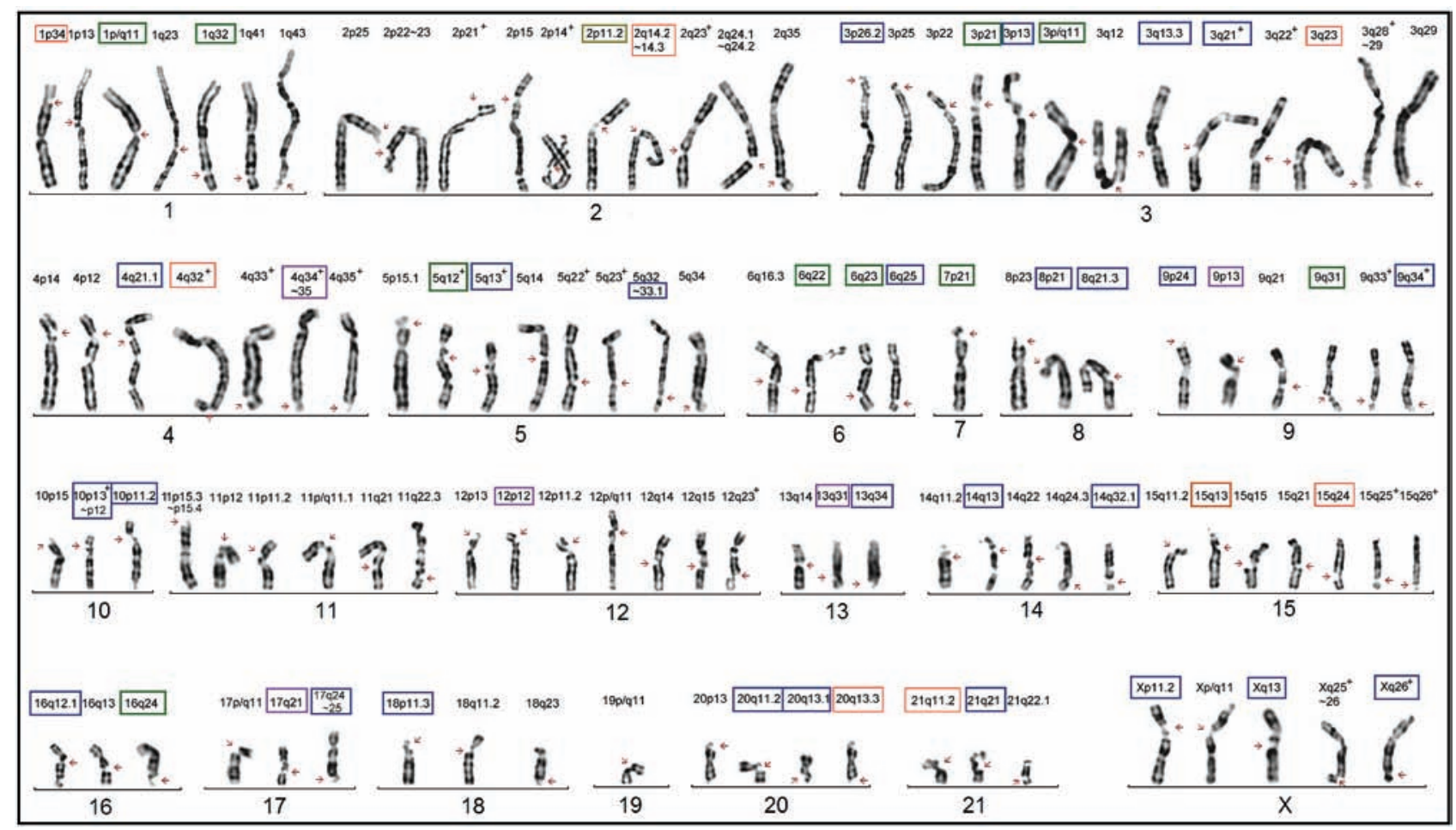

Figure 2. Cytogenetic detection of new aphidicolin-induced FS. Also shown are FS that were not included in the genome browser but described before. For each site the cytoband is given and an arrow indicates the FS. +, FS that might be larger and fused with a neighbor FS; green frame, taken from (37); blue frame, taken from (19); olive frame, taken from (34); brown frame, taken from (38); purple frame, taken from (29); red frame, single break events in the current study.

added to the existing 88 genome browser entries (Table I and Fig. 3).

\section{Discussion}

Frequency of FS. The common classification of FS is based on their induction with specific chemicals or frequencies in the population. The latter is divided into common and rare FS. Common FS are thought to be part of the normal chromosome structure and can be expressed in every individual (11). Nevertheless, they were also differentially expressed between different individuals, ethnic groups, sex and inducibility $(12,19,20,23,24,25)$. According to the literature, the most frequently-detected FS are FRA3B (3p14.2) and FRA16D (16q23.2), which was confirmed in the current study. Even within the three investigated individuals the frequencies for those two FS were almost the same. Contrary, it is known that not all common FS were inducible in every individual (26). This could also be shown in this study; although a huge number of metaphases were analyzed certain FS were exclusively observed only in one of the investigated test persons e.g. FRA17D (Table I). Additionally, most FS vary in their inter-individual frequency; examples are given in Fig. 1 and Table I. Only a few authors discuss the ethnic influence on FS expression (27). In this study proband III was CaucasianAsian descent whereas probands I and II were of Caucasian origin. Inter-individual frequency variations in FS-expression were also observed in other mammals like the mouse (28) and also between different tissues $(29,30)$. Although aphidicolin-induced FS were previously studied, for a closer 
Table I. Summary of all known and newly described aphidicolin-induced FS analyzed in 25,000 metaphase spreads with the extended nomenclature, cytogenetic localization, absolute numbers in different band levels (300-350, 400-450, 550-650, >650 bands per haploid karyotype) for three different subjects (I, II and III) and the frequency of every FS after aphidicolin induction in the current study.

\begin{tabular}{|c|c|c|c|c|c|c|c|c|c|c|c|c|c|c|c|}
\hline \multirow[b]{3}{*}{ All FS } & \multirow[b]{3}{*}{$\begin{array}{l}\text { Cytogenetic } \\
\text { localization }\end{array}$} & \multicolumn{12}{|c|}{ Number of FS per band level } & \multirow[b]{3}{*}{$\begin{array}{c}\sum \text { (all } \\
\text { subjects } \\
\text { and band } \\
\text { levels) }\end{array}$} & \multirow[b]{3}{*}{$\begin{array}{c}\text { Frequency } \\
\text { in \% (all } \\
\text { subjects an } \\
\text { band levels }\end{array}$} \\
\hline & & \multicolumn{3}{|c|}{$300-350$} & \multicolumn{3}{|c|}{$400-500$} & \multicolumn{3}{|c|}{$550-650$} & \multicolumn{3}{|c|}{$>650$} & & \\
\hline & & III & I & II & III & I & II & III & I & II & III & I & II & & \\
\hline FRA1A & $1 \mathrm{p} 36$ & 10 & 2 & 2 & 25 & 17 & 7 & 21 & 15 & 6 & 2 & 2 & 1 & 110 & 0.488 \\
\hline FRA1 $^{\text {h }}$ & $1 \mathrm{p} 34$ & - & - & - & - & - & 1 & - & - & - & - & - & - & 1 & 0.004 \\
\hline FRA1B & $1 \mathrm{p} 32$ & 25 & 5 & 1 & 81 & 55 & 38 & 51 & 37 & 26 & 7 & 5 & 5 & 336 & 1.491 \\
\hline FRA1L & $1 \mathrm{p} 31$ & 9 & 3 & 1 & 18 & 28 & 8 & 8 & 20 & 9 & 2 & 1 & 1 & 108 & 0.479 \\
\hline FRA1D & $1 \mathrm{p} 22$ & - & - & - & - & - & - & - & 1 & - & 1 & - & - & 2 & 0.009 \\
\hline FRA1M ${ }^{\mathrm{d}}$ & $1 \mathrm{p} 21.3$ & 55 & 7 & 13 & 178 & 118 & 75 & 73 & 116 & 83 & 10 & 12 & 11 & 751 & 3.333 \\
\hline FRA1E & $1 \mathrm{p} 21.2$ & - & - & - & 5 & 2 & 1 & 2 & 3 & 1 & 1 & - & - & 15 & 0.067 \\
\hline FRA1N & $1 \mathrm{p} 13$ & 2 & - & - & 8 & 1 & - & 1 & - & 1 & - & - & - & 13 & 0.058 \\
\hline $\mathrm{FRA} \mathrm{O}^{\mathrm{e}}$ & 1p11/q11 & 4 & 3 & 4 & 20 & 23 & 9 & 8 & 15 & 9 & - & 1 & 3 & 99 & 0.439 \\
\hline FRA1Ja & $1 \mathrm{q} 12$ & 2 & - & - & 8 & 5 & 1 & 3 & 6 & - & - & 1 & 1 & 27 & 0.120 \\
\hline FRA1F & $1 \mathrm{q} 21$ & - & 1 & - & 4 & 7 & 5 & 1 & 3 & 1 & - & - & - & 22 & 0.098 \\
\hline FRA1P & $1 q 23$ & 1 & - & 1 & 3 & - & - & 1 & - & 1 & - & 1 & - & 8 & 0.036 \\
\hline FRA1G & $1 \mathrm{q} 25.1$ & 17 & 2 & 2 & 39 & 25 & 26 & 14 & 26 & 19 & 2 & 4 & 4 & 180 & 0.799 \\
\hline FRA1K & $1 \mathrm{q} 31$ & 5 & - & - & 7 & 2 & 4 & 4 & 7 & 1 & - & 1 & - & 31 & 0.138 \\
\hline FRA1Q $^{\mathrm{e}}$ & $1 \mathrm{q} 32$ & 1 & - & - & 9 & 5 & 4 & 1 & 2 & - & - & - & - & 22 & 0.098 \\
\hline FRA1R & $1 q 41$ & 2 & 2 & - & 3 & 4 & - & 4 & 1 & 1 & 1 & - & 1 & 19 & 0.084 \\
\hline FRA1H $^{a}$ & $1 \mathrm{q} 42$ & 1 & 1 & - & 6 & 3 & 1 & 9 & 5 & 1 & 1 & 1 & - & 29 & 0.129 \\
\hline FRA1S & $1 q 43$ & - & - & - & 1 & 1 & - & 1 & - & - & - & - & - & 3 & 0.013 \\
\hline FRA1I & $1 \mathrm{q} 44$ & 16 & 10 & 2 & 84 & 97 & 52 & 39 & 115 & 74 & 2 & 9 & 18 & 518 & 2.299 \\
\hline FRA2M & $2 p 25$ & - & - & 2 & 7 & 4 & 1 & 2 & 6 & 3 & - & - & 1 & 26 & 0.115 \\
\hline FRA2C & $2 \mathrm{p} 24.2$ & 19 & 7 & 4 & 65 & 99 & 25 & 24 & 50 & 27 & 8 & 7 & 6 & 341 & 1.513 \\
\hline FRA2N & $2 p 22-23$ & 1 & - & - & 7 & 5 & 2 & 3 & 4 & 2 & - & - & - & 24 & 0.107 \\
\hline FRA2O & $2 p 21$ & - & 1 & - & 1 & 1 & - & 1 & 3 & 1 & 1 & - & - & 9 & 0.040 \\
\hline FRA2D & $2 \mathrm{p} 16.2$ & 38 & 11 & 6 & 158 & 93 & 27 & 53 & 80 & 39 & 4 & 6 & 9 & 524 & 2.325 \\
\hline FRA2P & $2 p 15$ & - & - & - & 4 & - & - & 2 & - & 2 & 3 & - & - & 11 & 0.049 \\
\hline FRA2Q & $2 p 14$ & - & - & - & 4 & - & - & 2 & - & - & - & - & - & 6 & 0.027 \\
\hline FRA2E & $2 \mathrm{p} 13$ & 3 & 2 & - & 14 & 11 & 5 & 5 & 6 & 5 & - & 1 & 1 & 53 & 0.235 \\
\hline FRA2L ${ }^{\mathrm{i}}$ & $2 \mathrm{p} 11.2$ & - & - & - & 3 & - & 4 & 1 & - & 4 & - & - & 1 & 13 & 0.058 \\
\hline FRA2R & $2 \mathrm{p} 11 / 2 \mathrm{q} 11$ & 3 & 2 & 2 & 11 & 6 & 2 & 6 & 5 & 2 & - & - & 1 & 40 & 0.178 \\
\hline FRA2A ${ }^{d}$ & $2 q 11.2$ & - & - & - & 11 & - & 4 & 1 & 4 & - & - & - & - & 20 & 0.089 \\
\hline $\mathrm{FRA} \mathrm{B}^{\mathrm{d}}$ & $2 q 13$ & - & - & - & 4 & 3 & 3 & 1 & 1 & 3 & - & - & - & 15 & 0.067 \\
\hline FRA2 $^{\text {h }}$ & $2 q 14.2-14.3$ & - & - & - & - & - & - & - & - & 1 & - & - & - & 1 & 0.004 \\
\hline FRA2F & $2 q 21.3$ & 12 & 4 & 1 & 49 & 60 & 24 & 19 & 33 & 16 & - & 3 & 3 & 224 & 0.994 \\
\hline FRA2K $^{\mathrm{d}}$ & $2 q 22.3$ & 15 & 5 & 2 & 33 & 22 & 11 & 13 & 17 & 20 & 2 & - & 1 & 141 & 0.626 \\
\hline FRA2S & $2 q 23$ & 4 & - & 1 & 8 & 4 & 3 & 2 & 1 & - & - & - & - & 23 & 0.102 \\
\hline FRA2T & $2 q 24$ & 1 & - & - & 1 & 4 & - & - & 4 & 1 & - & - & 1 & 12 & 0.053 \\
\hline FRA2G & $2 q 31$ & 8 & 1 & 2 & 16 & 8 & 4 & 11 & 11 & 2 & 1 & 1 & 4 & 69 & 0.306 \\
\hline FRA2H & $2 q 32.1$ & 69 & 29 & 17 & 223 & 182 & 74 & 73 & 139 & 49 & 10 & 9 & 6 & 880 & 3.905 \\
\hline FRA2I & $2 q 33$ & 27 & 11 & 6 & 75 & 104 & 27 & 28 & 73 & 25 & 5 & 1 & 6 & 388 & 1.722 \\
\hline FRA2U & $2 q 35$ & - & 1 & 1 & 5 & 7 & - & 3 & 3 & 2 & - & - & 1 & 23 & 0.102 \\
\hline FRA2J & $2 q 37.3$ & 9 & 2 & 5 & 39 & 22 & 9 & 24 & 18 & 9 & 3 & 4 & 4 & 148 & 0.657 \\
\hline FRA3E ${ }^{g}$ & $3 \mathrm{p} 26$ & 3 & - & 1 & 13 & 13 & 8 & 5 & 14 & 12 & 1 & 1 & - & 71 & 0.315 \\
\hline
\end{tabular}


Table I. Continued.

\begin{tabular}{|c|c|c|c|c|c|c|c|c|c|c|c|c|c|c|c|}
\hline \multirow[b]{3}{*}{ All FS } & \multirow[b]{3}{*}{$\begin{array}{l}\text { Cytogenetic } \\
\text { localization }\end{array}$} & \multicolumn{12}{|c|}{ Number of FS per band level } & \multirow[b]{3}{*}{$\begin{array}{c}\sum \text { (all } \\
\text { subjects } \\
\text { and band } \\
\text { levels) }\end{array}$} & \multirow[b]{3}{*}{$\begin{array}{c}\text { Frequency } \\
\text { in \% (all } \\
\text { subjects and } \\
\text { band levels) }\end{array}$} \\
\hline & & \multicolumn{3}{|c|}{$300-350$} & \multicolumn{3}{|c|}{$400-500$} & \multicolumn{3}{|c|}{$550-650$} & \multicolumn{3}{|c|}{$>650$} & & \\
\hline & & III & $\mathrm{I}$ & II & III & I & II & III & I & II & III & $\mathrm{I}$ & II & & \\
\hline FRA3F & $3 p 25$ & 4 & 2 & 3 & 7 & 9 & 10 & 6 & 3 & 4 & 2 & 2 & 1 & 53 & 0.235 \\
\hline FRA3A & $3 \mathrm{p} 24.2$ & 21 & 10 & 2 & 137 & 35 & 19 & 24 & 29 & 11 & 8 & 2 & 1 & 299 & 1.327 \\
\hline FRA3G & $3 p 22$ & - & - & - & - & - & - & - & 1 & 2 & 1 & - & - & 4 & 0.018 \\
\hline $\mathrm{FRA} 3 \mathrm{H}^{\mathrm{e}}$ & $3 \mathrm{p} 21$ & 2 & 1 & - & - & 10 & 5 & 2 & 9 & 3 & - & - & 2 & 34 & 0.151 \\
\hline FRA3B & $3 p 14.2$ & 202 & 71 & 53 & 682 & 751 & 256 & 289 & 544 & 233 & 36 & 24 & 48 & 3189 & 14.153 \\
\hline FRA3 I ${ }^{\mathrm{g}}$ & $3 p 13$ & 2 & 1 & - & 11 & 7 & 4 & 4 & 5 & 3 & 1 & - & 1 & 39 & 0.173 \\
\hline FRA3J & $3 p / q 11$ & - & - & - & 1 & 5 & 3 & 4 & 3 & - & - & - & - & 16 & 0.071 \\
\hline FRA3K & $3 q 12$ & - & - & - & - & 1 & 2 & - & 1 & 1 & - & - & - & 5 & 0.022 \\
\hline FRA3L ${ }^{g}$ & $3 q 13.3$ & 6 & 1 & 4 & 13 & 28 & 12 & 14 & 24 & 6 & 1 & 2 & 2 & 113 & 0.501 \\
\hline FRA3M ${ }^{g}$ & $3 q 21$ & - & - & - & 5 & - & - & 2 & 3 & - & - & - & - & 10 & 0.044 \\
\hline FRA3N & $3 q 22$ & - & - & - & 3 & 2 & 1 & 2 & 3 & - & 1 & - & - & 12 & 0.053 \\
\hline FRA3 ${ }^{\text {h }}$ & $3 q 23$ & - & - & - & - & - & - & 1 & - & - & - & - & - & 1 & 0.004 \\
\hline FRA3D & $3 q 25$ & 3 & 3 & 2 & 43 & 15 & 10 & 12 & 22 & 9 & 2 & 1 & 1 & 123 & 0.546 \\
\hline FRA3Of $^{f}$ & $3 q 26$ & - & - & - & - & - & - & - & - & - & - & - & - & 0 & 0.000 \\
\hline FRA3C & $3 q 27$ & 12 & 8 & 4 & 50 & 45 & 14 & 24 & 45 & 24 & 5 & 4 & 4 & 239 & 1.061 \\
\hline FRA3P & $3 q 28-29$ & - & 1 & 1 & 3 & 1 & 1 & 2 & - & - & - & - & 1 & 10 & 0.044 \\
\hline FRA3Q & $3 q 29$ & - & - & - & 2 & - & 1 & - & 1 & - & - & - & - & 4 & 0.018 \\
\hline FRA4A & $4 p 16.1$ & 8 & 1 & 4 & 35 & 32 & 8 & 8 & 20 & 12 & 3 & 3 & 4 & 138 & 0.612 \\
\hline FRA4D & $4 \mathrm{p} 15$ & 1 & 2 & - & 8 & 5 & - & 3 & 2 & 2 & - & 1 & 1 & 25 & 0.111 \\
\hline FRA4G & $4 p 14$ & - & - & - & - & - & 2 & 1 & - & 1 & - & - & - & 4 & 0.018 \\
\hline FRA4H & $4 p 12$ & 1 & - & - & 1 & 2 & - & - & 2 & - & - & - & - & 6 & 0.027 \\
\hline FRA4B $^{b}$ & $4 q 12$ & 1 & 1 & - & 9 & 5 & 4 & 3 & 5 & 2 & 1 & - & 1 & 32 & 0.142 \\
\hline FRA4I $^{g}$ & $4 q 21$ & 4 & 1 & 2 & 23 & 4 & 17 & 10 & 4 & 13 & - & 1 & 2 & 81 & 0.359 \\
\hline FRA4Fi & $4 q 22$ & 27 & 5 & 6 & 35 & 34 & 11 & 11 & 17 & 6 & 1 & 1 & - & 154 & 0.683 \\
\hline FRA4J & $4 q 23$ & - & - & - & - & - & - & - & - & - & - & - & - & 0 & 0.000 \\
\hline FRA4E & $4 q 27$ & 14 & 4 & 3 & 25 & 14 & 8 & 6 & 6 & 4 & - & 2 & - & 86 & 0.382 \\
\hline FRA4C & $4 q 31.1$ & 34 & 25 & 6 & 95 & 135 & 41 & 19 & 92 & 28 & 2 & 8 & 5 & 490 & 2.175 \\
\hline FRA4 ${ }^{\text {h }}$ & $4 q 32$ & - & - & - & - & - & - & - & 1 & - & - & - & - & 1 & 0.004 \\
\hline FRA4K & $4 q 33$ & - & - & 1 & 3 & 1 & - & 3 & - & - & - & - & - & 8 & 0.036 \\
\hline FRA4L $^{f}$ & $4 q 34-35$ & - & - & - & 1 & 1 & - & - & 3 & - & - & - & - & 5 & 0.022 \\
\hline FRA4M & $4 q 35$ & 1 & - & 1 & 2 & 1 & - & 1 & 1 & 1 & - & - & 1 & 9 & 0.040 \\
\hline FRA5H & $5 p 15$ & - & - & 2 & 8 & 7 & 2 & 3 & 6 & 1 & - & - & - & 29 & 0.129 \\
\hline FRA5E & $5 p 14$ & 7 & 3 & 1 & 6 & 22 & 6 & 4 & 7 & 4 & 1 & 3 & 2 & 66 & 0.293 \\
\hline FRA5A & $5 p 13$ & 2 & 2 & 1 & 13 & 8 & 6 & 1 & 7 & 3 & - & - & - & 43 & 0.191 \\
\hline FRA5I & $5 \mathrm{p} 11 / 5 \mathrm{q} 11$ & 7 & 2 & 1 & 19 & 11 & 7 & 9 & 12 & 6 & 1 & 1 & 1 & 77 & 0.342 \\
\hline FRA5J & $5 q 12$ & - & - & - & 3 & 1 & 1 & 2 & - & 1 & - & - & 1 & 9 & 0.040 \\
\hline FRA5K ${ }^{\mathrm{g}}$ & $5 q 13$ & - & 1 & - & - & 6 & 3 & - & 6 & 3 & - & - & - & 19 & 0.084 \\
\hline FRA5L & $5 q 14$ & - & - & - & 4 & 3 & 2 & 1 & 6 & 1 & - & 1 & - & 18 & 0.080 \\
\hline FRA5D & $5 q 15$ & 13 & 11 & 10 & 33 & 59 & 24 & 13 & 20 & 5 & - & - & - & 188 & 0.834 \\
\hline FRA5F & $5 q 21$ & 5 & 2 & 3 & 24 & 15 & 23 & 8 & 12 & 18 & 1 & 1 & 4 & 116 & 0.515 \\
\hline FRA5M & $5 q 22$ & - & - & - & 5 & 1 & 1 & 1 & - & 1 & - & - & - & 9 & 0.040 \\
\hline FRA5N & $5 q 23$ & - & - & 1 & 1 & 1 & - & 2 & 1 & - & 1 & - & - & 7 & 0.031 \\
\hline FRA5C & $5 q 31.1$ & 2 & 2 & 1 & 19 & 19 & 8 & 4 & 19 & 6 & 1 & 2 & 1 & 84 & 0.373 \\
\hline $\mathrm{FRA} \mathrm{O}_{\mathrm{g}}$ & $5 q 33$ & - & - & - & - & - & 1 & - & 1 & 1 & - & - & 1 & 4 & 0.018 \\
\hline FRA5P & $5 q 34$ & - & - & - & 4 & 1 & 1 & 4 & 1 & - & - & - & - & 11 & 0.049 \\
\hline
\end{tabular}


Table I. Continued.

\begin{tabular}{|c|c|c|c|c|c|c|c|c|c|c|c|c|c|c|c|}
\hline \multirow[b]{3}{*}{ All FS } & \multirow[b]{3}{*}{$\begin{array}{l}\text { Cytogenetic } \\
\text { localization }\end{array}$} & \multicolumn{12}{|c|}{ Number of FS per band level } & \multirow[b]{3}{*}{$\begin{array}{c}\sum \text { (all } \\
\text { subjects } \\
\text { and band } \\
\text { levels) }\end{array}$} & \multirow[b]{3}{*}{$\begin{array}{l}\text { Frequency } \\
\text { in \% (all } \\
\text { subjects and } \\
\text { band levels }\end{array}$} \\
\hline & & \multicolumn{3}{|c|}{$300-350$} & \multicolumn{3}{|c|}{$400-500$} & \multicolumn{3}{|c|}{$550-650$} & \multicolumn{3}{|c|}{$>650$} & & \\
\hline & & III & I & II & III & I & II & III & I & II & III & I & II & & \\
\hline FRA5G & $5 q 35$ & 1 & 1 & - & 6 & 6 & 3 & 1 & 4 & 3 & - & 2 & & 27 & 0.120 \\
\hline FRA6B & $6 \mathrm{p} 25.1$ & 7 & 5 & 5 & 30 & 41 & 32 & 12 & 36 & 42 & 3 & 2 & 7 & 222 & 0.985 \\
\hline FRA6A $^{\mathrm{d}}$ & $6 \mathrm{p} 23$ & - & - & - & - & - & - & - & 2 & - & - & - & - & 2 & 0.009 \\
\hline FRA6C & $6 \mathrm{p} 22.2$ & - & - & - & 2 & 1 & 1 & 1 & 4 & 5 & - & - & - & 14 & 0.062 \\
\hline FRA6H ${ }^{\mathrm{i}}$ & $6 \mathrm{p} 21.1$ & 1 & - & - & 1 & 4 & 3 & 1 & 7 & 5 & - & - & - & 22 & 0.098 \\
\hline FRA6I & $6 \mathrm{p} 11 / \mathrm{q} 11$ & 1 & 2 & 1 & 7 & 4 & 4 & 6 & 7 & 2 & 1 & 1 & 1 & 37 & 0.164 \\
\hline FRA6D $^{\mathrm{b}}$ & $6 q 13$ & 2 & 1 & - & - & 1 & - & 1 & 2 & - & - & - & - & 7 & 0.031 \\
\hline FRA6G & $6 q 15$ & 3 & - & 2 & 5 & 3 & 2 & 2 & 3 & 4 & - & - & 2 & 26 & 0.115 \\
\hline FRA6J & $6 q 16.3$ & 3 & - & - & 9 & 2 & - & 3 & - & 2 & - & - & - & 19 & 0.084 \\
\hline FRA6F & $6 \mathrm{q} 21$ & 15 & 7 & 2 & 30 & 27 & 11 & 7 & 22 & 7 & 8 & - & 1 & 137 & 0.608 \\
\hline FRA6K ${ }^{\mathrm{e}}$ & $6 \mathrm{q} 22$ & 1 & 2 & - & 8 & 8 & 7 & 4 & 8 & 10 & 2 & - & 2 & 52 & 0.231 \\
\hline FRA6L $\mathrm{L}^{\mathrm{e}}$ & $6 q 23$ & 1 & 1 & 2 & 10 & 8 & 2 & 4 & 1 & 1 & - & - & 1 & 31 & 0.138 \\
\hline FRA6M ${ }^{\mathrm{g}}$ & $6 q 25$ & 1 & 1 & 2 & 6 & 4 & 4 & 2 & 4 & 1 & - & 2 & 1 & 28 & 0.124 \\
\hline FRA6E & $6 \mathrm{q} 26$ & 50 & 21 & 5 & 155 & 153 & 19 & 56 & 128 & 25 & 10 & 4 & 10 & 636 & 2.823 \\
\hline FRA7B & $7 \mathrm{p} 22$ & 3 & 2 & 1 & 41 & 21 & 8 & 16 & 30 & 37 & 3 & 3 & 15 & 180 & 0.799 \\
\hline FRA7L ${ }^{e}$ & $7 \mathrm{p} 21$ & 2 & - & - & 3 & 1 & 1 & - & - & - & - & & & 7 & 0.031 \\
\hline FRA7C & $7 \mathrm{p} 14.2$ & 7 & 3 & 2 & 26 & 33 & 13 & 10 & 21 & 4 & 1 & 1 & 1 & 122 & 0.541 \\
\hline FRA7D & $7 \mathrm{p} 13$ & 2 & 2 & - & 35 & 23 & 10 & 15 & 27 & 6 & - & 3 & 1 & 124 & 0.550 \\
\hline FRA7A $^{\mathrm{d}}$ & $7 \mathrm{p} 11.2$ & 1 & - & - & 3 & 1 & - & 4 & - & 2 & - & 2 & - & 13 & 0.058 \\
\hline FRA7J & $7 \mathrm{q} 11.23$ & 19 & 8 & 2 & 41 & 49 & 22 & 10 & 26 & 6 & 1 & - & 3 & 187 & 0.830 \\
\hline FRA7E & $7 \mathrm{q} 21.2$ & 6 & 1 & 2 & 45 & 11 & 8 & 23 & 7 & 7 & 1 & 2 & - & 113 & 0.501 \\
\hline FRA7F & $7 \mathrm{q} 22$ & 1 & - & 1 & 7 & 5 & 4 & 4 & 1 & 1 & - & - & - & 24 & 0.107 \\
\hline FRA7K ${ }^{\mathrm{i}}$ & 7q22-31.1 & 36 & 21 & 13 & 111 & 160 & 81 & 42 & 100 & 49 & 7 & 9 & 6 & 635 & 2.818 \\
\hline FRA7G & $7 q 31.2$ & 2 & - & 1 & 4 & 4 & - & 4 & 3 & 5 & - & 1 & 1 & 25 & 0.111 \\
\hline FRA7H & $7 \mathrm{q} 32.3$ & 20 & 11 & 5 & 104 & 91 & 73 & 33 & 104 & 67 & 7 & 8 & 12 & 535 & 2.374 \\
\hline FRA7M & $7 \mathrm{q} 34$ & 7 & 2 & 2 & 26 & 7 & 17 & 21 & 11 & 14 & 2 & - & 1 & 110 & 0.488 \\
\hline FRA7I & $7 \mathrm{q} 36$ & 1 & - & - & 6 & 5 & 2 & 1 & 3 & 1 & - & - & - & 19 & 0.084 \\
\hline FRA8G & $8 p 23$ & 1 & - & - & 4 & 3 & - & 2 & 4 & $\mathbf{1}$ & - & - & - & 15 & 0.067 \\
\hline FRA8H ${ }^{g}$ & $8 \mathrm{p} 21$ & - & - & 1 & 3 & 1 & 2 & 1 & 1 & - & - & - & - & 9 & 0.040 \\
\hline FRA8I & $8 p 11 / q 11$ & - & 1 & - & 8 & 5 & 9 & 4 & 7 & 3 & - & - & 4 & 41 & 0.182 \\
\hline FRA8F & $8 \mathrm{q} 13$ & - & - & - & - & - & - & - & 1 & - & - & - & - & 1 & 0.004 \\
\hline FRA8J" & $8 \mathrm{q} 21.3$ & - & - & - & 3 & - & - & 2 & 2 & - & - & - & - & 7 & 0.031 \\
\hline FRA8B & $8 \mathrm{q} 22.1$ & 20 & 1 & 1 & 52 & 27 & 30 & 23 & 34 & 14 & - & - & 6 & 208 & 0.923 \\
\hline FRA8A ${ }^{d}$ & $8 \mathrm{q} 22.3$ & - & - & - & 2 & - & 1 & - & 1 & - & - & - & - & 4 & 0.018 \\
\hline FRA8C & $8 \mathrm{q} 24.1$ & 9 & 3 & 3 & 28 & 30 & 20 & 23 & 27 & 23 & 4 & 2 & 8 & 180 & 0.799 \\
\hline FRA8D & $8 \mathrm{q} 24.3$ & 3 & - & - & 10 & 2 & 4 & 3 & 8 & 8 & 2 & 1 & - & 41 & 0.182 \\
\hline FRA9H ${ }^{g}$ & 9 p24 & - & - & - & - & 2 & 1 & 2 & 2 & - & - & - & - & 7 & 0.031 \\
\hline FRA9Gi & $9 \mathrm{p} 22$ & 1 & - & - & 4 & - & 1 & 2 & - & 1 & - & - & - & 9 & 0.040 \\
\hline FRA9A $^{d}$ & $9 \mathrm{p} 21$ & - & 1 & - & 4 & 7 & 3 & 2 & 5 & - & 1 & - & - & 23 & 0.102 \\
\hline FRA9I $^{\mathrm{f}}$ & $9 \mathrm{p} 13$ & - & - & - & - & - & 1 & 1 & - & 1 & - & - & - & 3 & 0.013 \\
\hline FRA9Fa & $9 q 12$ & 6 & - & 2 & 13 & 22 & 11 & 14 & 18 & 9 & 4 & 2 & 1 & 102 & 0.453 \\
\hline FRA9Je & $9 \mathrm{q} 13$ & - & - & - & - & - & - & - & - & - & - & - & - & 0 & 0,000 \\
\hline FRA9K & $9 q 21$ & 1 & 1 & - & 2 & 4 & 3 & 1 & 2 & 3 & - & - & - & 17 & 0.075 \\
\hline FRA9D & $9 q 22.1$ & 4 & 1 & 1 & 11 & 15 & 4 & 3 & 12 & 1 & - & - & - & 52 & 0.231 \\
\hline FRA9L $^{\mathrm{e}}$ & $9 q 31$ & 2 & - & - & 1 & - & 1 & 2 & 1 & 1 & - & - & - & 8 & 0.036 \\
\hline
\end{tabular}


Table I. Continued.

\begin{tabular}{|c|c|c|c|c|c|c|c|c|c|c|c|c|c|c|c|}
\hline \multirow[b]{3}{*}{ All FS } & \multirow[b]{3}{*}{$\begin{array}{l}\text { Cytogenetic } \\
\text { localization }\end{array}$} & \multicolumn{12}{|c|}{ Number of FS per band level } & \multirow[b]{3}{*}{$\begin{array}{c}\sum \text { (all } \\
\text { subjects } \\
\text { and band } \\
\text { levels) }\end{array}$} & \multirow[b]{3}{*}{$\begin{array}{c}\text { Frequency } \\
\text { in \% (all } \\
\text { subjects and } \\
\text { band levels) }\end{array}$} \\
\hline & & \multicolumn{3}{|c|}{$300-350$} & \multicolumn{3}{|c|}{$400-500$} & \multicolumn{3}{|c|}{$550-650$} & \multicolumn{3}{|c|}{$>650$} & & \\
\hline & & III & I & II & III & $\mathrm{I}$ & II & III & I & II & III & I & II & & \\
\hline FRA9B & $9 q 32$ & 22 & 5 & 3 & 93 & 65 & 24 & 44 & 49 & 28 & 5 & - & 3 & 341 & 1.513 \\
\hline FRA9M & $9 q 33$ & 1 & - & - & 2 & - & 2 & - & 2 & 2 & - & - & - & 9 & 0.040 \\
\hline FRA9N ${ }^{g}$ & $9 q 34$ & - & 1 & 1 & 2 & 5 & 4 & 3 & 2 & 4 & - & 1 & - & 23 & 0.102 \\
\hline FRA10H & 10p15 & 2 & 1 & 1 & 4 & 4 & 4 & 1 & 1 & 3 & - & - & 1 & 22 & 0.098 \\
\hline FRA10Ig & $10 p 12-13$ & 2 & 1 & 1 & 14 & 1 & 3 & 2 & 2 & - & 1 & - & - & 27 & 0.120 \\
\hline FRA10Jg & $10 p 11.2$ & 2 & - & - & 10 & 2 & 1 & 3 & 4 & 2 & - & - & - & 24 & 0.107 \\
\hline FRA10G & $10 \mathrm{q} 11.2$ & - & 1 & - & 9 & 9 & 1 & 4 & 6 & 2 & - & - & - & 32 & 0.142 \\
\hline FRA10C ${ }^{b}$ & $10 q 21$ & - & - & - & 7 & 4 & 2 & 1 & 5 & - & - & - & 1 & 20 & 0.089 \\
\hline FRA10D & $10 \mathrm{q} 22.1$ & 7 & 3 & 1 & 23 & 16 & 5 & 5 & 6 & 3 & - & - & - & 69 & 0.306 \\
\hline FRA10A ${ }^{a}$ & $10 \mathrm{q} 23.3$ & 1 & - & 2 & 7 & 3 & 2 & 8 & 2 & 2 & - & - & 1 & 28 & 0.124 \\
\hline FRA $10 K^{d}$ & $10 \mathrm{q} 24.2$ & 2 & - & - & 7 & 5 & - & 5 & 3 & - & - & - & - & 22 & 0.098 \\
\hline FRA10E & $10 \mathrm{q} 25.2$ & - & - & 1 & 10 & 7 & 8 & 6 & 7 & 2 & - & - & 2 & 43 & 0.191 \\
\hline FRA10F & 10q26.1 & 5 & 1 & 1 & 32 & 11 & 4 & 20 & 10 & 3 & 2 & 1 & 2 & 92 & 0.408 \\
\hline FRA11J & 11p15.3-p15.4 & 1 & - & - & 1 & 3 & - & 1 & 1 & 1 & - & - & - & 8 & 0.036 \\
\hline FRA11C & $11 \mathrm{p} 15.1$ & 6 & 5 & - & 23 & 25 & 13 & 10 & 22 & 3 & 2 & 1 & 5 & 115 & 0.510 \\
\hline FRA11D & $11 \mathrm{p} 14.2$ & 17 & 5 & 1 & 53 & 40 & 13 & 22 & 34 & 14 & 4 & 1 & 5 & 209 & 0.928 \\
\hline FRA11E & $11 \mathrm{p} 13$ & 6 & 3 & 4 & 28 & 32 & 7 & 17 & 29 & 8 & - & 3 & 3 & 140 & 0.621 \\
\hline FRA11K & $11 p 12$ & - & - & - & 2 & - & - & - & - & - & - & - & - & 2 & 0.009 \\
\hline FRA11L & $11 p 11.2$ & - & - & - & 2 & 1 & 1 & - & 1 & 1 & - & - & - & 6 & 0.027 \\
\hline FRA11M & 11p11/q11 & 1 & - & 1 & 8 & 7 & 2 & 7 & 3 & 2 & 2 & - & 2 & 35 & 0.155 \\
\hline FRA11H & $11 q 13.3$ & - & 1 & 1 & 2 & 7 & - & 2 & 2 & 2 & - & - & - & 17 & 0.075 \\
\hline FRA11F & $11 \mathrm{q} 14.2$ & 24 & 12 & 9 & 122 & 64 & 30 & 38 & 55 & 31 & 7 & 6 & 1 & 399 & 1.771 \\
\hline FRA11N & $11 q 21$ & - & - & - & 1 & 5 & 1 & 1 & 8 & - & - & 1 & - & 17 & 0.075 \\
\hline FRA110 & $11 q 22$ & 1 & - & - & 3 & - & 1 & 2 & 2 & - & - & - & - & 9 & 0.040 \\
\hline FRA11G & $11 \mathrm{q} 23.3$ & 1 & 1 & 1 & 4 & 5 & - & 4 & 2 & 2 & 1 & 1 & - & 22 & 0.098 \\
\hline FRA12F & $12 p 13$ & 1 & - & - & 1 & 2 & - & 1 & - & 1 & - & - & - & 6 & 0.027 \\
\hline FRA12G ${ }^{\mathrm{f}}$ & $12 \mathrm{p} 12$ & - & - & - & 2 & 4 & - & 2 & - & 1 & 1 & - & - & 10 & 0.044 \\
\hline FRA12H & $12 \mathrm{p} 11.2$ & 1 & 1 & - & - & 1 & 2 & - & - & - & - & - & - & 5 & 0.022 \\
\hline FRA12I & 12p11/q11 & 1 & - & - & 2 & 6 & - & 1 & 4 & - & - & - & 2 & 16 & 0.071 \\
\hline FRA12A & $12 \mathrm{q} 13.1$ & 1 & - & - & 1 & 4 & 3 & 1 & 2 & 4 & 1 & - & - & 17 & 0.075 \\
\hline FRA12J & $12 q 14$ & - & - & - & - & - & - & - & 1 & 2 & - & - & 1 & 4 & 0.018 \\
\hline FRA12K & $12 q 15$ & - & 1 & - & 2 & - & - & - & 3 & - & 2 & 1 & - & 9 & 0.040 \\
\hline FRA12B & $12 q 21.3$ & 7 & - & 1 & 26 & 8 & 6 & 13 & 10 & 6 & 2 & 1 & 2 & 82 & 0.364 \\
\hline FRA12L & $12 q 23.1$ & 3 & - & - & 7 & - & 2 & 3 & 6 & 5 & - & - & - & 26 & 0.115 \\
\hline FRA12E & $12 q 24$ & 4 & 1 & 3 & 12 & 14 & 11 & 6 & 6 & 6 & 1 & - & 1 & 65 & 0.288 \\
\hline FRA13A & $13 q 13.2$ & 15 & 7 & 2 & 63 & 45 & 23 & 28 & 32 & 19 & 2 & 1 & 2 & 239 & 1.061 \\
\hline FRA13G & $13 q 14$ & 1 & - & 1 & 3 & 1 & - & 1 & 1 & - & - & - & - & 8 & 0.036 \\
\hline FRA13C & $13 q 21.2$ & 1 & - & - & 6 & 2 & 6 & 4 & 5 & 2 & - & - & 2 & 28 & 0.124 \\
\hline FRA $13 E^{i}$ & $13 q 22$ & - & 1 & - & 4 & 4 & 2 & 2 & 6 & 2 & - & - & - & 21 & 0.093 \\
\hline FRA13H ${ }^{\mathrm{f}}$ & $13 q 31$ & - & - & - & - & - & 2 & - & - & 1 & - & - & - & 3 & 0.013 \\
\hline FRA13D & $13 q 32$ & 1 & - & 2 & 6 & 10 & 3 & 4 & 7 & 3 & - & 1 & 3 & 40 & 0.178 \\
\hline FRA13I & $13 q 34$ & 3 & - & 1 & 10 & 4 & 3 & 9 & 6 & 7 & 1 & 1 & 1 & 46 & 0.204 \\
\hline FRA14D & $14 q 11.2$ & - & - & - & - & 1 & - & - & 1 & 1 & - & - & - & 3 & 0.013 \\
\hline FRA14Eg & $14 q 13$ & 1 & - & - & 9 & 6 & 4 & 5 & 8 & 4 & 1 & - & 1 & 39 & 0.173 \\
\hline FRA14A & $14 q 21.2$ & 3 & - & 1 & 9 & 4 & 3 & 3 & 4 & 3 & - & - & 1 & 31 & 0.138 \\
\hline
\end{tabular}


Table I. Continued.

\begin{tabular}{|c|c|c|c|c|c|c|c|c|c|c|c|c|c|c|c|}
\hline \multirow[b]{3}{*}{ All FS } & \multirow[b]{3}{*}{$\begin{array}{l}\text { Cytogenetic } \\
\text { localization }\end{array}$} & \multicolumn{12}{|c|}{ Number of FS per band level } & \multirow[b]{3}{*}{$\begin{array}{c}\sum \text { (all } \\
\text { subjects } \\
\text { and band } \\
\text { levels) }\end{array}$} & \multirow[b]{3}{*}{$\begin{array}{c}\text { Frequency } \\
\text { in \% (all } \\
\text { subjects and } \\
\text { band levels) }\end{array}$} \\
\hline & & \multicolumn{3}{|c|}{$300-350$} & \multicolumn{3}{|c|}{$400-500$} & \multicolumn{3}{|c|}{$550-650$} & \multicolumn{3}{|c|}{$>650$} & & \\
\hline & & III & $\mathrm{I}$ & II & III & I & II & III & I & II & III & I & II & & \\
\hline FRA14F & $14 q 22$ & - & - & - & 7 & 3 & 3 & - & 1 & 3 & 1 & - & 1 & 19 & 0.084 \\
\hline FRA14B & $14 \mathrm{q} 23$ & 6 & 5 & 4 & 39 & 54 & 45 & 23 & 87 & 35 & 4 & 7 & 13 & 322 & 1.429 \\
\hline FRA14C & $14 \mathrm{q} 24.1$ & 4 & 3 & 3 & 12 & 20 & 18 & 8 & 9 & 8 & 1 & - & - & 86 & 0.382 \\
\hline FRA14G & $14 \mathrm{q} 24.3$ & - & - & - & 1 & - & - & - & 1 & - & - & - & - & 2 & 0.009 \\
\hline FRA14Hg & $14 q 32$ & - & 1 & - & 4 & - & 3 & - & 1 & - & - & - & - & 9 & 0.040 \\
\hline FRA15C & $15 q 11.2$ & - & - & - & 1 & - & 1 & - & - & 1 & - & - & - & 3 & 0.013 \\
\hline FRA $15 B^{\mathrm{i}}$ & $15 \mathrm{q} 13$ & 1 & 1 & - & 6 & 2 & - & - & 1 & - & - & - & 1 & 12 & 0.053 \\
\hline FRA15D & $15 q 15$ & 2 & - & - & 4 & 9 & 2 & - & 2 & 1 & - & - & - & 20 & 0.089 \\
\hline FRA15E & $15 q 21$ & - & - & - & - & 1 & 2 & - & 1 & 1 & - & - & - & 5 & 0.022 \\
\hline FRA15A & $15 \mathrm{q} 22$ & - & 1 & - & 5 & 2 & 5 & 1 & - & 1 & 1 & - & 2 & 18 & 0.080 \\
\hline FRA15 $^{\text {h }}$ & $15 q 24$ & - & - & - & - & - & $\mathbf{1}$ & - & - & - & - & - & - & 1 & 0.004 \\
\hline FRA15F & $15 q 25$ & - & - & - & 2 & - & - & 1 & 1 & 1 & - & - & - & 5 & 0.022 \\
\hline FRA15G & $15 q 26$ & - & - & - & - & 1 & - & - & 1 & 1 & - & - & - & 3 & 0.013 \\
\hline FRA16A ${ }^{d}$ & $16 \mathrm{p} 13.11$ & - & - & - & - & 2 & 1 & 1 & 4 & 1 & - & 1 & 2 & 12 & 0.053 \\
\hline FRA16E $\mathrm{E}^{\mathrm{c}}$ & $16 \mathrm{p} 12.1$ & 1 & - & - & - & 1 & 1 & - & 1 & - & - & 1 & 1 & 6 & 0.027 \\
\hline FRA16F & $16 \mathrm{p} 11 / 16 q 11$ & - & - & - & 3 & 4 & 3 & 2 & 1 & 1 & - & - & 1 & 15 & 0.067 \\
\hline FRA16G $\mathrm{G}^{\mathrm{g}}$ & $16 q 12$ & - & - & - & - & 1 & 1 & - & 2 & - & - & 1 & - & 5 & 0.022 \\
\hline FRA16H & $16 q 13$ & - & - & - & 2 & - & - & - & - & - & - & - & - & 2 & 0.099 \\
\hline FRA16 ${ }^{e}$ & $16 \mathrm{q} 21$ & - & - & - & 1 & - & - & - & 1 & - & - & - & - & 2 & 0.099 \\
\hline FRA16C & $16 \mathrm{q} 22.1$ & 4 & 5 & - & 14 & 18 & 17 & 9 & 23 & 15 & 1 & 3 & 1 & 110 & 0.488 \\
\hline FRA16D & $16 \mathrm{q} 23.2$ & 78 & 36 & 18 & 283 & 443 & 110 & 133 & 381 & 146 & 17 & 26 & 36 & 1707 & 7.576 \\
\hline FRA16Je & $16 \mathrm{q} 24$ & - & - & - & 3 & 3 & - & - & 1 & 2 & - & 2 & 1 & 12 & 0.053 \\
\hline FRA $17 A^{c}$ & $17 \mathrm{p} 12$ & - & - & - & 2 & - & 4 & 2 & 2 & 1 & - & - & - & 11 & 0.049 \\
\hline FRA17C & 17p11/17q11 & - & - & - & 1 & 2 & - & 1 & 4 & - & - & - & - & 8 & 0.036 \\
\hline FRA17D ${ }^{f}$ & $17 \mathrm{q} 21$ & - & - & - & 1 & - & - & - & - & - & - & - & - & 1 & 0.004 \\
\hline FRA17B & $17 \mathrm{q} 23.1$ & - & - & - & 4 & 4 & 1 & 2 & 2 & 1 & 1 & 1 & - & 16 & 0.071 \\
\hline FRA17E & $17 q 24-25$ & 3 & - & - & 10 & 12 & 4 & 5 & 9 & 8 & 1 & 2 & - & 54 & 0.240 \\
\hline FRA18Dg & $18 \mathrm{p} 11.3$ & - & - & - & 3 & 3 & 1 & - & 5 & 1 & - & - & 1 & 14 & 0.062 \\
\hline FRA18E & $18 q 11.2$ & - & - & - & $\mathbf{1}$ & 1 & $\mathbf{1}$ & 1 & 2 & - & - & 1 & 1 & 8 & 0.036 \\
\hline FRA18A & $18 \mathrm{q} 12.2$ & 2 & 1 & 5 & 29 & 18 & 3 & 18 & 23 & 8 & - & - & - & 107 & 0.475 \\
\hline FRA18B & $18 \mathrm{q} 21.3$ & 3 & 2 & 1 & 7 & 7 & 6 & 3 & 8 & 4 & - & - & - & 41 & 0.182 \\
\hline FRA18C $\mathrm{C}^{\mathrm{i}}$ & $18 \mathrm{q} 22$ & 1 & - & 1 & 2 & 2 & 1 & 3 & 1 & 4 & - & - & - & 15 & 0.067 \\
\hline FRA18F & $18 q 23$ & 2 & - & - & 1 & 2 & 2 & 1 & 2 & - & - & - & 1 & 11 & 0.049 \\
\hline FRA19B $^{d}$ & $19 \mathrm{p} 13.1$ & - & - & - & 1 & 3 & - & 2 & - & 2 & - & - & - & 8 & 0.036 \\
\hline FRA19C & 19p11/q11 & - & - & - & - & - & 1 & - & 1 & - & - & - & 1 & 3 & 0.013 \\
\hline FRA19A $^{a}$ & $19 \mathrm{q} 13$ & - & - & - & 3 & 3 & 1 & 2 & 2 & - & - & - & 1 & 12 & 0.053 \\
\hline FRA20C & $20 p 13$ & - & - & - & 2 & 7 & 4 & - & 9 & 1 & - & - & 1 & 24 & 0.107 \\
\hline FRA20B & $20 \mathrm{p} 12.2$ & 1 & - & - & 7 & 10 & 8 & 4 & 13 & 10 & - & - & 2 & 55 & 0.244 \\
\hline FRA20A ${ }^{d}$ & $20 \mathrm{p} 11.23$ & - & - & - & - & 4 & 1 & - & - & 1 & - & - & - & 6 & 0.027 \\
\hline FRA20Dg & $20 \mathrm{q} 11.2$ & - & - & - & 1 & 2 & - & 1 & 1 & - & - & - & - & 5 & 0.022 \\
\hline FRA20E $\mathrm{E}^{\mathrm{g}}$ & $20 \mathrm{q} 13.1$ & 1 & - & - & 3 & 4 & 2 & 3 & 5 & 1 & 3 & - & 1 & 23 & 0.102 \\
\hline FRA20 & $20 q 13.3$ & - & - & - & - & - & - & - & 1 & - & - & - & - & 1 & 0.004 \\
\hline FRA21 $^{\mathrm{h}}$ & $21 q 11.2$ & - & - & - & - & 1 & - & - & - & - & - & - & - & 1 & 0.004 \\
\hline FRA21 $\mathrm{A}^{\mathrm{g}}$ & $21 \mathrm{q} 21$ & - & - & - & 1 & 1 & - & 1 & 2 & - & - & - & - & 5 & 0.022 \\
\hline FRA21B & $21 q 22.1$ & 1 & - & - & 3 & 1 & 1 & - & 1 & 3 & - & 1 & - & 11 & 0.049 \\
\hline
\end{tabular}


Table I. Continued.

\begin{tabular}{|c|c|c|c|c|c|c|c|c|c|c|c|c|c|c|c|}
\hline \multirow[b]{3}{*}{ All FS } & \multirow[b]{3}{*}{$\begin{array}{l}\text { Cytogenetic } \\
\text { localization }\end{array}$} & \multicolumn{11}{|c|}{ Number of FS per band level } & & \multirow[b]{3}{*}{$\begin{array}{c}\sum \text { (all } \\
\text { subjects } \\
\text { and band } \\
\text { levels) }\end{array}$} & \multirow[b]{3}{*}{$\begin{array}{l}\text { Frequency } \\
\text { in \% (all } \\
\text { subjects and } \\
\text { band levels) }\end{array}$} \\
\hline & & & -3 & & & -5 & & & -65 & & & $>650$ & & & \\
\hline & & $\overline{\text { III }}$ & I & II & $\overline{\text { III }}$ & I & $\overline{\text { II }}$ & III & I & $\overline{\mathrm{II}}$ & III & $\mathrm{I}$ & $\overline{\text { II }}$ & & \\
\hline
\end{tabular}

\begin{tabular}{|c|c|c|c|c|c|c|c|c|c|c|c|c|c|c|c|}
\hline FRA22B & $22 q 12.2$ & 2 & - & 2 & 12 & 10 & 4 & 5 & 13 & 11 & - & . & 3 & 62 & 0.275 \\
\hline FRA22A ${ }^{d}$ & $22 q 13.2$ & 3 & - & - & 17 & 10 & 4 & 5 & 28 & 5 & 1 & - & 1 & 74 & 0.328 \\
\hline FRAXB & Xp22.31 & 70 & 24 & 30 & 261 & 267 & 130 & 93 & 201 & 116 & 13 & 13 & 20 & 1238 & 5.494 \\
\hline $\mathrm{FRAXG}^{\mathrm{g}}$ & Xp11.2 & - & - & - & 1 & - & - & - & 1 & - & - & - & - & 2 & 0.009 \\
\hline FRAXH & Xp11/q11 & - & 1 & - & 7 & 5 & - & 4 & 9 & 2 & 1 & 2 & - & 31 & 0.138 \\
\hline FRAXI $^{\mathrm{g}}$ & $\mathrm{Xq} 13$ & - & - & 2 & 11 & 6 & 2 & 2 & 8 & 4 & 1 & - & 1 & 37 & 0.164 \\
\hline FRAXC & Xq22.1 & 31 & 13 & 8 & 109 & 90 & 40 & 40 & 83 & 44 & 6 & 3 & 11 & 478 & 2.121 \\
\hline FRAXJ & $\mathrm{Xq} 25$ & 1 & - & - & 3 & 1 & - & 1 & 2 & 1 & 1 & - & - & 10 & 0.044 \\
\hline FRAXK ${ }^{g}$ & Xq26 & - & - & - & - & 1 & 1 & - & - & - & - & - & - & 2 & 0.009 \\
\hline FRAXD & Xq27.2 & 2 & 1 & 1 & 7 & 17 & 2 & 2 & 14 & - & 1 & - & - & 47 & 0.209 \\
\hline FRAXE & $\mathrm{Xq} 28$ & - & - & - & - & 1 & - & - & 1 & - & - & 1 & 1 & 4 & 0.018 \\
\hline$\sum($ all FS $)$ & & 1344 & 516 & 358 & 4978 & 4625 & 2032 & 2059 & 3806 & 1871 & 282 & 260 & 406 & 22537 & 0.276 \\
\hline
\end{tabular}

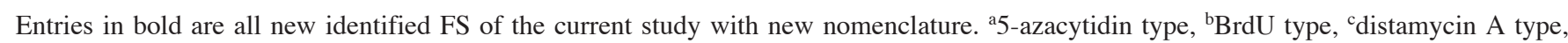
${ }^{\mathrm{d}}$ folic acid type, ${ }^{\mathrm{e}}(37)$ with new nomenclature, ${ }^{\mathrm{f}}(23)$ with new nomenclature, ${ }^{\mathrm{g}}(19)$ with new nomenclature, ${ }^{\mathrm{h}}$ new identified FS but only one time recorded and not included in the nomenclature, ${ }^{\mathrm{F}} \mathrm{FS}$ that were already assigned to the nomenclature by other authors but not included in genome browsers.

Table II. Number of FS and average distance in Mb between FS for single chromosomes.

\begin{tabular}{lcccccccccccc}
\hline Chromosome & 1 & 2 & 3 & 4 & 5 & 6 & 7 & 8 & 9 & 10 & 11 & 12 \\
\hline $\begin{array}{l}\text { Number of FS } \\
\text { Interval in Mb }\end{array}$ & 14 & 21 & 17 & 13 & 15 & 13 & 13 & 9 & 12 & 10 & 12 & 10 \\
& 14.53 & 11.52 & 11.76 & 14.69 & 12.07 & 13.15 & 12.23 & 16.22 & 11.67 & 13.35 & 11.17 & 13.20 \\
\hline Chromosome & 13 & 14 & 15 & 16 & 17 & 18 & 19 & 20 & 21 & 22 & $\mathrm{X}$ & $\mathrm{Y}$ \\
\hline $\begin{array}{l}\text { Number of FS } \\
\text { Interval in Mb }\end{array}$ & 14 & 8 & 7 & 9 & 5 & 6 & 3 & 5 & 2 & 2 & 9 & 1 \\
\hline
\end{tabular}

look on inter-individual and/or ethnic influences on FS frequency variation, larger population studies on comparable conditions would be needed.

New FS. The current study identified 61 until now not reported FS and 7 unique break events that were not reported before. The frequency of these new sites varies between $0.009 \%$ (FRA14G) and $0.231 \%$ (FRA3E). Other, wellestablished aphidicolin-induced sites range from $0.067 \%$ (FRA1E) to $14.153 \%$ (FRA3B), indicating that the newly described sites have a low expression rate and therefore were not detected or reported previously. Although some authors stress the necessity for fragility to be defined as a statistical phenomenon (e.g. 12,31), the main part of fragile sites is expressed in low frequencies, sometimes limited to single individuals and depends on several other factors such as aphidicolin concentration, gender or ethnic origin. Furthermore, complicating is the definition of a fragile site since several authors use different definitions or count also single breaks as fragile sites $(11,30,32)$. Therefore, we decided to use a more practical definition where all sites are included that were observed more than once in this study or were already published elsewhere to minimize false positive random background breaks.

Only little is known about the influence of local chromatin structure for FS expression. Obviously FS are overrepresented in GTG light bands. This holds also true for the new identified FS, 59\% of which appears in GTG-light bands. Recently, an unusual chromatin structure or failure of chromatin condensation was postulated as inductors of FS (33). 

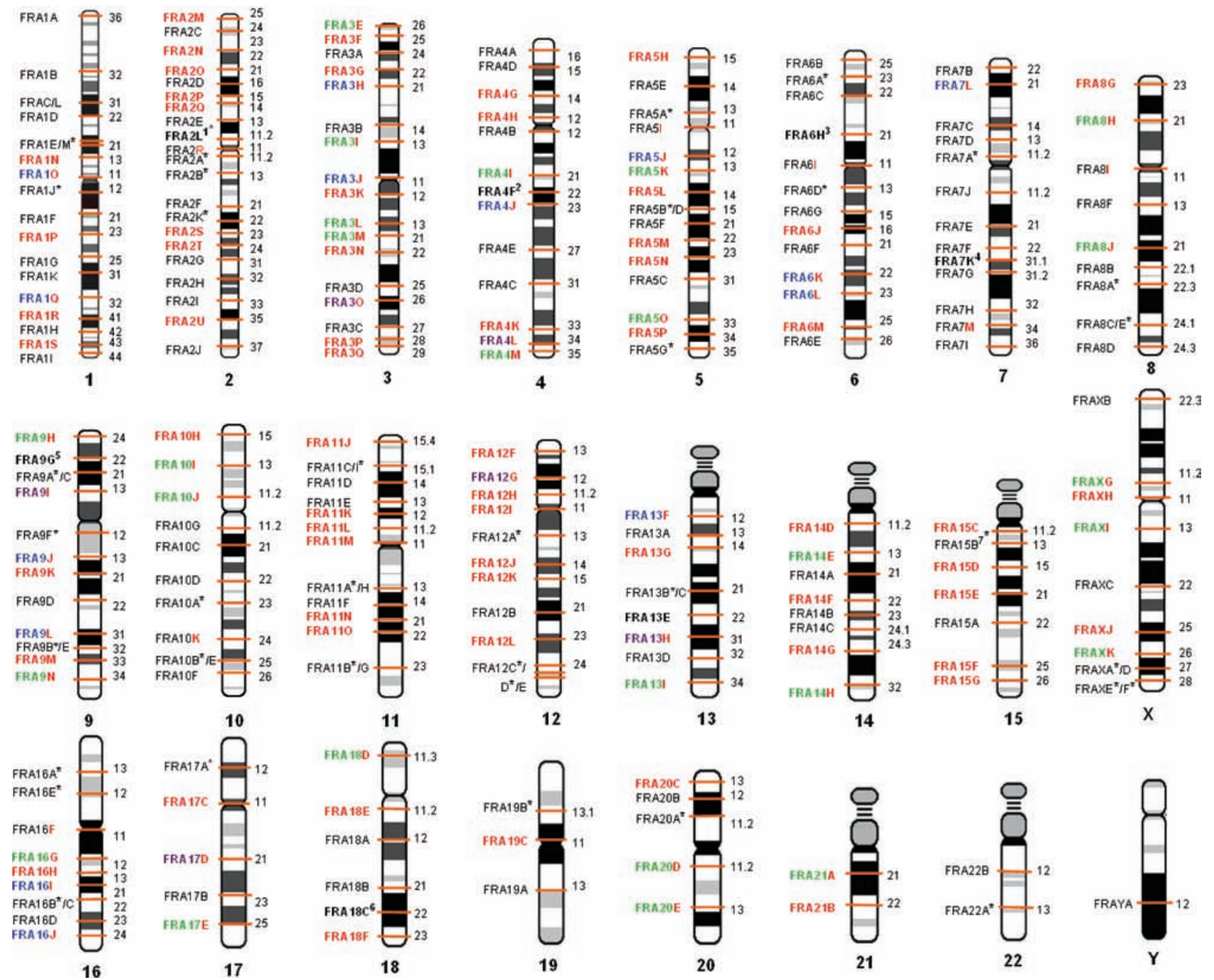

Figure 3. Overview of all known and newly described FS (orange line in ideograms). Left to the ideograms is shown the FS nomenclature, to the right the cytoband. Bold text FS are not listed in NCBI 36.3 genome browser; black, are NCBI 36.3 listed FS; red, are newly described FS in the current study; blue, taken from (37); green, taken from (19); light blue, taken from (19); purple, taken from (23); "FS not reported as aphidicolin type before; 1: (34), 2: (13), 3: (15), 4: (14), 5: (16), 6: (17), 7: (38).

Table III. Comparison of FS, not reported as aphidicolin inducible before, with literature data. ${ }^{\mathrm{a}}$

\begin{tabular}{lcccccc}
\hline FS & & \multicolumn{5}{c}{ Frequency in $(\%)$} \\
\cline { 5 - 7 } & $\begin{array}{c}\text { Cytogenetic } \\
\text { localization }\end{array}$ & $\begin{array}{c}\text { Frequency in the } \\
\text { current study }(\%) \\
\mathrm{n}=25,000\end{array}$ & $\begin{array}{c}\text { Manjunatha } \text { et al, } \\
2002(39) \\
\mathrm{n}=100\end{array}$ & $\begin{array}{c}\text { Takahashi } \text { et al, } \\
1988(40) \\
\mathrm{n}=?\end{array}$ & $\begin{array}{c}\text { Rao } \text { et al, } \\
1988(32) \\
\mathrm{n}=800\end{array}$ & $\begin{array}{c}\text { Tastemir } \text { et al } \\
2006(41) \\
\mathrm{n}=1,443\end{array}$ \\
\hline FRA1Q & $1 \mathrm{q} 32$ & 0.098 & - & - & - & 1.68 \\
FRA2A & $2 \mathrm{q} 11.2$ & 0.089 & 1.0 & - & - & - \\
FRA5J & $5 \mathrm{q} 12$ & 0.040 & - & - & 0.4 & - \\
FRA10C & $10 \mathrm{q} 21$ & 0.089 & - & - & - & - \\
FRA17A & $17 \mathrm{p} 12$ & 0.049 & - & -1 & - & - \\
FRAXK & Xq26 & 0.009 & - & - & - & 0.33 \\
\hline
\end{tabular}

a(39): 5-fluorodeoxyuridin (FUdR); (40): distamycin A; (32): FUdR; (41): folate absence; $\mathrm{n}$ = number of studied metaphases. 
Nomenclature. The common nomenclature system for FS includes the abbreviation FRA followed by chromosome number and a letter starting with A arranged in a linear order from pter to qter. Chromosome and chromatide breaks in this study were counted as new FS and included in the nomenclature when they were observed more than one time. Seven unique breaks were also recorded (Fig. 2 and Table I), but not included in the nomenclature (Fig. 3 and Table I).

The current study identified 230 compared to only 129 rare and common FS, which are listed in the NCBI genome browser. Even more complicating is the fact that 15 of these sites map at the same cytogenetic region but have different names because of different ways of induction (NCBI genome browser, Fig. 3). Additionally, 52 identified and in parts mapped FS were already published, but not included in the current genome browser versions $(13,14,15,16,17,19,23,34)$. Most of these sites were confirmed in this study which underlines their eligibility to be appreciated as FS.

FS classification. FS are commonly classified in rare FS that can be induced by folate absence, distamycin A, 5-fluorodeoxyuridin or BrdU, and in common FS inducible by aphidicolin, BrdU or 5-azacytidine. In the current study exclusively aphidicolin induction was used, and surprisingly nearly every type of rare and common FS could be recorded. Overall, this makes the classification in rare and common FS at least questionable. The main reason to explain this surprising finding might be a relative small amount of FS studied in near-comparable studies. Differences to literature data were also observed in the frequency of single sites when the induction was done in a different way (Table III).

Based on frequency differences within expression rates of so-called common FS from $14.153 \%$ (FRA3B, this study) to $0.004 \%$ (e.g. FRA17D, this study) some authors $(12,24,28)$ suggested a classification in high (HFFS) and low frequency FS (LFFS). This classification seems to be more reliable than the historical division in common and rare FS. Also a classification based on the chemical induction of FS seems questionable; particularly as in this study every type of FS could be induced by aphidicolin.

Conclusion. Location of FS seems to be independent of the chemical agents used. Thus, a classification in different induction types is no longer warranted. According to Hecht et al (4) and others we prefer a classification of high and low frequency FS. In order to prevent double naming of FS in the same cytogenetic location, caused historically by different modes of induction, an expanded definition of FS into fragile regions is helpful as it becomes more and more clear from molecular mapping data that especially aphidicolin inducible FS do not break at defined sequences but in breakage-prone regions up to $10 \mathrm{Mb}(35,36)$ where the break is most likely to appear.

\section{Acknowledgments}

Supported by the Evangelische Studienwerk e.V. Villigst and in parts by DFG (WE3617/2-1, LI820/11-1, 436 RUS 17/ 135/03, 436 RUS 17/109/04, 436 RUS 17/22/06), Boehringer Ingelheim Fonds, IZKF Jena (Start-up S16), IZKF MD-
Fellowships, Prochance 2008, Stefan Morsch Stiftung and Stiftung Leukämie. We thank T. Lehmann (Jena University Hospital, Institute of Medical Statistics, Informatics and Documentation) for helpful discussion.

\section{References}

1. Dekaban A: Persisting clone of cells with an abnormal chromosome in a woman previously irradiated. J Nucl Med 10: 740-746, 1965.

2. Magenis RE, Hecht F and Lovrien EW: Heritable fragile site on chromosome 16: probable localization of haptoglobin locus in man. Science 170: 85-87, 1970.

3. Berger R, Bloomfield CD and Sutherland GR: Report of the committee on chromosome rearrangements in neoplasia and on fragile sites (Human Gene Mapping 8, Helsinki Conference). Cytogenet Cell Genet 40: 490-535, 1985.

4. Hecht F: Rare, polymorphic, and common fragile sites: a classification. Hum Genet 74: 207-208, 1986.

5. Durkin SG and Glover TW: Chromosome fragile sites. Annu Rev Genet 41: 169-192, 2007.

6. Sutherland GR: Fragile sites on human chromosomes: demonstration of their dependence on the type of tissue culture medium. Science 197: 265-266, 1977.

7. Lukusa T and Fryns JP: Human chromosome fragility. Biochim Biophys Acta 1779: 3-16, 2008.

8. Mitelman F: Recurrent chromosome aberrations in cancer. Mutat Res 462: 247-253, 2000.

9. Gümüs G, Sunguroglu A, Tükün A, Sayin DB and Bökesoy I: Common fragile sites associated with the breakpoints of chromosomal aberrations in hematologic neoplasms. Cancer Genet Cytogenet 133: 168-171, 2002.

10. Schoder C, Liehr T, Velleuer E, Wilhelm K, Blaurock N, Weise A and Mrasek K: New aspects on chromosomal instability: Chromosomal break-points in Fanconi anemia patients colocalize on the molecular level with fragile sites. Int J Oncol 36: 307-312, 2010.

11. Craig-Holmes AP, Strong LC, Goodacre A and Pathak S: Variation in the expression of aphidicolin-induced fragile sites in human lymphocyte cultures. Hum Genet 76: 134-137, 1987.

12. Denison SR, Simper RK and Greenbaum IF: How common are common fragile sites in humans: interindividual variation in the distribution of aphidicolin-induced fragile sites. Cytogenet Genome Res 101: 8-16, 2003.

13. Rozier L, El-Achkar E, Apiou F and Debatisse M: Characterization of a conserved aphidicolin-sensitive common fragile site at human $4 \mathrm{q} 22$ and mouse $6 \mathrm{C} 1$ : possible association with an inherited disease and cancer. Oncogene 23: 6872-6880, 2004.

14. Helmrich A, Stout-Weider K, Matthaei A, Hermann K, Heiden T and Schrock E: Identification of the human/mouse syntenic common fragile site FRA7K/Fra12C1-relation of FRA7K and other human common fragile sites on chromosome 7 to evolutionary breakpoints. Int J Cancer 120: 48-54, 2007.

15. Fechter A, Buettel I, Kuehnel E, Schwab M and Savelyeva L: Cloning of genetically tagged chromosome break sequences reveals new fragile sites at $6 \mathrm{p} 21$ and $13 \mathrm{q} 22$. Int J Cancer 120: 2359-2367, 2007.

16. Sawinska M, Schmitt JG, Sagulenko E, Westermann F, Schwab M and Savelyeva L: Novel aphidicolin-inducible common fragile site FRA9G maps to 9p22.2, within the C9orf39 gene. Genes Chromosomes Cancer 46: 991-999, 2007.

17. Debacker K, Winnepenninckx B, Ben-Porat N, Fitz Patrick D, Van Luijk R, Scheers S, Kerem B and Frank Kooy R: FRA18C: a new aphidicolin-inducible fragile site on chromosome 18q22, possibly associated with in vivo chromosome breakage. J Med Genet 44: 347-352, 2007.

18. Wyandt HE and Tonk VS: Atlas of Human Chromosome Heteromorphisms. Kluwer Academic Publishers, Dordrecht, 2004.

19. Simonic I and Gericke GS: The enigma of common fragile sites. Hum Genet 97: 524-531, 1996.

20. Glover TW, Berger C, Coyle J and Echo B: DNA polymerase alpha inhibition by aphidicolin induces gaps and breaks at common fragile sites in human chromosomes. Hum Genet 67 : 136-142, 1984.

21. Verma RS and Babu A: Human chromosomes - manual of basic techniques. Pergamon Press, New York, Oxford, 1989. 
22. Shaffer LG, Slovak ML, Campbell LJ (eds): ISCN: An International System for Human Cytogenetic Nomenclature. S. Karger, Basel, 2009.

23. Kuwano A, Sugio Y, Murano I and Kajii T: Common fragile sites induced by folate deprivation, BrdU and aphidicolin: their frequency and distribution in Japanese individuals. Jinrui Idengaku Zasshi 33: 355-364, 1988.

24. Hecht F, Tajara EH, Lockwood D, Sandberg AA and Hecht BK: New common fragile sites. Cancer Genet Cytogenet 33: 1-9, 1988.

25. Tedeschi B, Vernole P, Sanna ML and Nicoletti B: Population cytogenetics of aphidicolin-induced fragile sites. Hum Genet 89: 543-547, 1992.

26. Sutherland GR, Baker E and Richards RI: Fragile sites still breaking. Trends Genet 14: 501-506, 1998.

27. Takahashi E, Hori T and Murata M: Population cytogenetics of rare fragile sites in Japan. Hum Genet 78: 121-126, 1988.

28. McAllister BF and Greenbaum IF: How common are common fragile sites: variation of aphidicolin-induced chromosomal fragile sites in a population of the deer mouse (Peromyscus maniculatus). Hum Genet 100: 182-188, 1997.

29. Kuwano A, Murano I and Kajii T: Cell type-dependent difference in the distribution and frequency of excess thymidine-induced common fragile sites: T lymphocytes and skin fibroblasts. Hum Genet 84: 527-531, 1990.

30. Murano I, Kuwano A and Kajii T: Fibroblast-specific common fragile sites induced by aphidicolin. Hum Genet 83: 45-48, 1989.

31. Greenbaum IF, Fulton JK, White ED and Dahm PF: Minimum sample sizes for identifying chromosomal fragile sites from individuals: Monte Carlo estimation. Hum Genet 101: 109-112, 1997.
32. Rao PN, Heerema NA and Palmer CG: Fragile sites induced by FUdR, caffeine, and aphidicolin. Their frequency, distribution, and analysis. Hum Genet 78: 21-26, 1988.

33. Wang YH: Chromatin structure of human chromosomal fragile sites. Cancer Lett 232: 70-78, 2006.

34. Schuffenhauer S, Lederer G and Murken J: A heritable folatesensitive fragile site on chromosome 2p 11.2 (FRA2L). Chromosome Res 4: 252-254, 1996.

35. Curatolo A, Limongi ZM, Pelliccia F and Rocchi A: Molecular characterization of the human common fragile site FRA1H. Genes Chromosomes Cancer 46: 487-493, 2007.

36. Zhu Y, McAvoy S, Kuhn R and Smith DI: RORA, a large common fragile site gene, is involved in cellular stress response. Oncogene 25: 2901-2908, 2006.

37. Borgaonkar DS: Chromosomal Variation in Man. 7th edition, Wiley-Liss, NY, 1994.

38. Karadeniz NN, Tunca Y and Imirzalioglu N: New heritable fragile site at $15 \mathrm{q} 13$ in both members of a nonconsanguineous couple. Am J Med Genet 118: 290-292, 2003.

39. Manjunatha KR, Chetan GK, Arathi R, Bhaskara Rao GV, Latha P, Padma S, Venkatesh HN, Srinath S, Girimaji SR and Sheshadri S: Frequency, association and Genetic implications of chromosomal fragile sites in mental retardation. IJHG 2: 33-39, 2002.

40. Takahashi E, Kaneko Y, Ishihara T, Minamihisamatsu M, Murata $\mathrm{M}$ and Hori T: A new rare distamycin A-inducible fragile site, fra(11) (p15.1), found in two acute nonlymphocytic leukemia (ANLL) patients with $\mathrm{t}(7 ; 11)(\mathrm{p} 15-\mathrm{p} 13 ; \mathrm{p} 15)$. Hum Genet 80: 124-126, 1988.

41. Tastemir D, Demirhan O and Sertdemir Y: Chromosomal fragile site expression in Turkish psychiatric patients. Psychiatry Res 144: 197-203, 2006. 